\title{
Discovery of novel anti-proliferative compounds against A549 cells by virtual screening
}

\author{
P Ajay Babu ${ }^{* 1,2}$, Mangamoori L Narasu², Srinivas Kolli ${ }^{3}$ \\ ${ }^{I}$ Bioinformatics Division, Translational Research Institute of Molecular Sciences (TRIMS), \\ 2-35-72, Sector-10, MVP colony, Visakhapatnam - 530 017, India \\ ${ }^{2}$ Centre for Biotechnology, Jawaharlal Nehru Technological University Hyderabad (JNTUH), \\ Kukatpally, Hyderabad-500085, India \\ ${ }^{3}$ Sri Venkateswara College of Pharmacy, Etcherla, Srikakulam-532402, India \\ *E-mail: ajay@trimslabs.com
}

(Received November 19,2009; accepted March 29,2010 ; published online April 23,2010)

\begin{abstract}
CDK2 (Cyclin Dependent Kinase 2) acts as a potential therapeutic target in cancer and several efforts have been made to find more specific, potent and selective ATP competitive CDK2 inhibitors. In this paper, we report a virtual screening approach that resulted in 54,558 Lipinski compliant hits from ZINC database based on the features exhibited by four compounds from our previous study. Docking and scoring of all compounds using GOLD (Genetic Optimisation for Ligand Docking) software, to evaluate the affinity of binding towards CDK2 enzyme 2UZO resulted in dock scores between $41.71-82.33 \mathrm{kcal} / \mathrm{mol}$. The resultant dataset of 392 hits were filtered based on the specificity between CDK2 and GSK-3 $\beta$ (Glycogen Synthase Kinase-3 $\beta$ ) to obtain 17 compounds that are more specific towards CDK2. Further, re-scoring of 17 best docked poses followed by a consensus scoring approach tested with five different scoring functions such as GOLD score, CHEM score implemented in GOLD 3.1, eHiTS_score (electronic High Throughput Screening), MolDock score of Molegro software and X-Score retrieved top hits. Finally, the top ten compounds were examined for anti-proliferative effects against human lung adenocarcinoma epithelial cell line, A549 using MTT assay.
\end{abstract}

Key Words: ZINC database, cross-docking, binding affinity, apoptosis, A549, MTT assay

Area of Interest: Virtual Screening and anti-cancer studies 


\section{Introduction}

Eukaryotic cell cycle progresses by transitions from one stage of the cell cycle to the next and are driven by phosphorylation events mediated by a family of serine/threonine protein kinases termed CDKs (Cyclin Dependent Kinases). These complexes are composed of the catalytic subunit $\mathrm{CDK}$ and the positive regulatory subunit cyclin [1]. Members of the CDK family are active at distinct points in the cell cycle. CDK2 is essential for G1 progression and S-phase entry and when complexed with cyclin E leads to phosphorylation on multiple sites of the retinoblastoma protein $(\mathrm{pRb})[2]$. Based on the studies that explored various CDK2 bound inhibitors and its role in cell cycle progression and proliferation, CDK2 acts as a potential therapeutic target in several proliferative diseases, including cancer [3]. Several kinds of compounds were reported as CDK2 inhibitors [4][5]. Though many of them possess inhibitory activities against CDK2, still many diverse compounds are being developed to increase potency and selectivity. These studies have provided the rationale to find new chemical entities with a reasonable inhibitory potency on CDK2 inhibitors.

Traditional synthesis of a series of new compounds utilizing combinatorial chemistry and highthroughput screening can be carried out at high cost and also are time consuming; whereas screening large databases of compounds can provide a feasible, alternative technique. Hence, in this paper we report screening a library of compounds from ZINC database [6] against CDK2 protein, 2UZO (PDB ID) complexed with a thiazolidinone inhibitor, extracted from protein data bank, by utilizing the docking software GOLD. In our previous work [7] we reported screening non-tested compounds for CDK2 inhibition based on the QSAR model and the biological activities are predicted. The molecules with enhanced predicted activity $(27 n, 41 n, 42 n$ and $43 n$ compounds) than any other compound from the data set are given in Figure 1 and hence a virtual screening approach was employed to filter the ZINC database towards extracting either similar or entirely diverse compounds based on the Lipinski features exhibited by these four compounds.

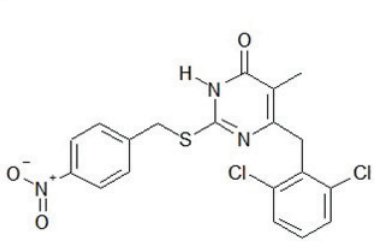

27n [Predicted $\log (1 / C): 8.93]$

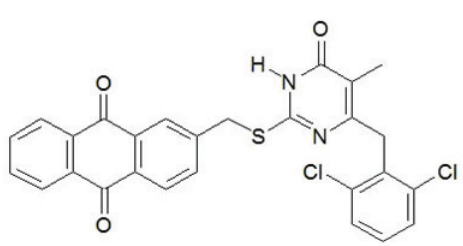

41n [Predicted $\log (1 / \mathrm{C}): 11.48]$

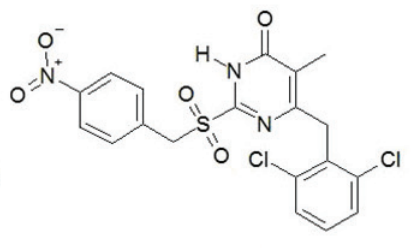

42n [Predicted $\log (1 / C): 9.07]$

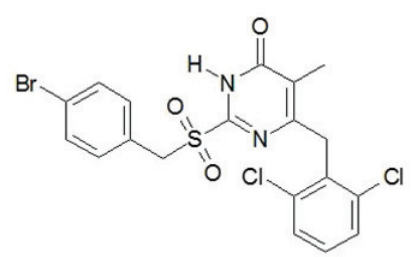

43n [Predicted $\log (1 / \mathrm{C}): 9.22]$

Figure 1. 2D structures of 27n, 41n, 42n and 43n compounds selected from our previous work [7] employing QSAR and virtual screening approach, used to screen ZINC database for diverse compounds. 


\section{Materials and Methods}

\subsection{Receptor X-ray structure}

The X-ray crystal structure of the CDK2 protein, 2UZO, in complex with a thiazolidinone inhibitor [8] C62 was recovered from Protein Data Bank (PDB: http://www.rcsb.org/pdb) and selected [19] as the receptor model in virtual screening program. We used the chemical compound library, ZINC database and the docking program GOLD [9] (Genetic Optimization for Ligand Docking) for virtual ligand docking and a consensus scoring and ranking was employed. Before screening ZINC database, the docking protocol was validated. 2UZO protein bound ligand was docked into the binding pocket to obtain the docked pose and the RMSD of all atoms between these two conformations was $0.83 \AA$ indicating that the parameters for docking simulation are good in reproducing the X-ray crystal structure. Therefore, the ZINC database was screened using molecular constraints option to retrieve compounds with similar or diverse structural features by taking mean Lipinski's values (data given in Table 1) of $\mathbf{2 7 n}, \mathbf{4 1} n, \mathbf{4 2 n}$ and $43 n$ compounds.

Table 1. Physico-chemical properties of $27 \mathrm{n}, 41 \mathrm{n}, \mathbf{4 2 n}$ and $43 \mathrm{n}$ compounds used to scan ZINC database, displaying predicted activities (log 1/C) and GOLD dock scores $(\mathrm{kcal} / \mathrm{mol})$.

\begin{tabular}{c|ccccccc}
\hline $\begin{array}{c}\text { Compound } \\
\text { Id }\end{array}$ & $\begin{array}{c}\text { Molecular } \\
\text { Weight }\end{array}$ & $\begin{array}{c}\text { H-bond } \\
\text { Acceptors }\end{array}$ & $\begin{array}{c}\text { H-bond } \\
\text { Donors }\end{array}$ & logP & $\begin{array}{c}\text { Rotatable } \\
\text { bonds }\end{array}$ & $\begin{array}{c}\text { Predicted } \\
\text { log (1/C) }\end{array}$ & $\begin{array}{c}\text { Dock Score } \\
\text { (kcal/mol) }\end{array}$ \\
\hline $\mathbf{2 7 n}$ & 436.33 & 4 & 1 & 4.86 & 0 & 8.93 & 55.16 \\
$\mathbf{4 1 n}$ & 521.43 & 4 & 1 & 5.31 & 2 & 11.48 & 64.88 \\
$\mathbf{4 2 n}$ & 468.33 & 6 & 1 & 3.92 & 0 & 9.07 & 56.29 \\
$\mathbf{4 3 n}$ & 502.22 & 4 & 1 & 4.76 & 1 & 9.22 & 57.74 \\
\hline from ref. [7] & & & & & & &
\end{tabular}

\subsection{Consensus Scoring and Ranking}

As docking and scoring play important roles in drug design, it has been reported that consensus scoring was generally more effective than single scoring for molecular docking and represented an effective way in getting improved hit rates in various virtual database screening studies [10][11][12]. In our study, we tested five different scoring functions such as GOLD score, CHEM score implemented in GOLD 3.1, eHiTS_score [13], MolDock score of Molegro software [14] and XScore [15]. Docking program GOLD was used to dock compounds and each scoring function was applied to generate classes. During ranking, signs of some scoring functions are changed to make certain that a lower score always indicates a higher affinity. 


\subsection{MTT assay}

MTT assay relies on the ability of live cells to reduce a water-soluble yellow dye MTT (3-(4,5Dimethylthiazol-2-yl)-2,5-diphenyltetrazolium bromide) to a water-insoluble purple formazan product. The MTT assay developed by Mosmann [16] was modified and used to determine the inhibitory effects of test compounds on growth of A549 cells in vitro. In brief, the trypsinized cells from T-25 flask were seeded in 96-well flat-bottomed tissue culture plate at a density of $5 \times 10^{3}$ cells/well in growth medium and cultured at $37^{\circ} \mathrm{C}$ in $5 \% \mathrm{CO}_{2}$ to adhere. After $48 \mathrm{hr}$ incubation, the cells were pretreated with growth medium and mixed with different concentrations of test compounds $(0.5,1,2,4,8,16,32$ and $64 \mu \mathrm{g} / \mathrm{ml})$ in triplicates to achieve a final volume of $100 \mu 1$ and then incubated for $48 \mathrm{hr}$. Compounds were prepared as $2 \mathrm{mg} / \mathrm{ml}$ concentration stock solutions in dimethyl sulfoxide (DMSO) and the final concentration of DMSO in the culture was within $0.2 \%$. Growth medium and solvent are used as controls. Each well then received $2 \mu 1$ of fresh MTT $\left(0.5 \mathrm{mg} / \mathrm{ml}\right.$ in PBS) followed by incubation for $2 \mathrm{hr}$ at $37^{\circ} \mathrm{C}$ The growth medium was removed from the wells and replaced with $100 \mu \mathrm{l}$ of DMSO to solubilize the colored formazan product. After 30 min incubation, the absorbance (Optical Density) of the culture plate was read at a wavelength of $570 \mathrm{~nm}$ on an ELISA reader, Thermo Electron Multiskan Spectrum spectrophotometer. The mean concentrations of each compound that generated $50 \%$ inhibitions $\left(\mathrm{IC}_{50}\right)$ were calculated using version 8 of Microcal Origin software. Mean OD values of each test compound were corrected by subtracting with the mean OD of blanks. Relative percent inhibition activity is expressed as:

$\%$ inhibition $=100-($ corrected mean OD of sample $\times 100 /$ corrected mean OD of control $)$

\section{Results and Discussion}

\subsection{Rationale to select $2 \mathrm{UZO}$ and $1 \mathrm{UV5}$ as potential targets}

It has been reported in literature that molecular docking studies on GSK-3 $\beta$ were carried out using X-ray crystal structure 1UV5 towards enrichment of inhibitors [17], pose prediction accuracy for enrichment of actives [18] etc. However, 2UZO needs to be justified and there were no reports in literature on molecular docking studies with 2UZO. Therefore, a search in PDB database revealed around $168 \mathrm{CDK} 2 \mathrm{X}$-ray crystallographic structures and about 20 CDK2 complexes were extracted to perform cross-docking studies, i.e. docking PDB ligands into non-native crystal structures of the target protein.

In this experiment, each ligand was docked into each of the protein structures using default parameters of GOLD. For this purpose, all CDK2 structures were superposed [19] with the first structure in the list, $1 \mathrm{AQ} 1$. Re-docking of all compounds into their respective native structures led 
to $\mathrm{RMSDs} \leq 2.5 \AA$ in 16 out of 20 cases $(80 \%)$. It was evident from the Table 2 that if any of the protein structures are considered (Best RMSD all ligands column), there was at least one good docking solution for each ligand. Moreover, when the number of best RMSDs obtained for each protein target against all ligands was considered, it was found that $2 \mathrm{UZO}$ outperform the task with ten best RMSDs and hence selected in screening and docking studies. Therefore, these results suggest that it would be beneficial to dock non-native ligands into many structures of the target, especially when the binding site residues are highly flexible, such as in CDKs.

Table 2. RMSD values of 20 CDK2 ligands in a cross-docking experiment Values in parenthesis represent resolution $(\AA)$. RMSD values more than $5.0 \AA$ are presented in dark background, RMSD between $5.0-2.5 \AA$ are in light grey color and RMSD less than $2.5 \AA$ are in white background.

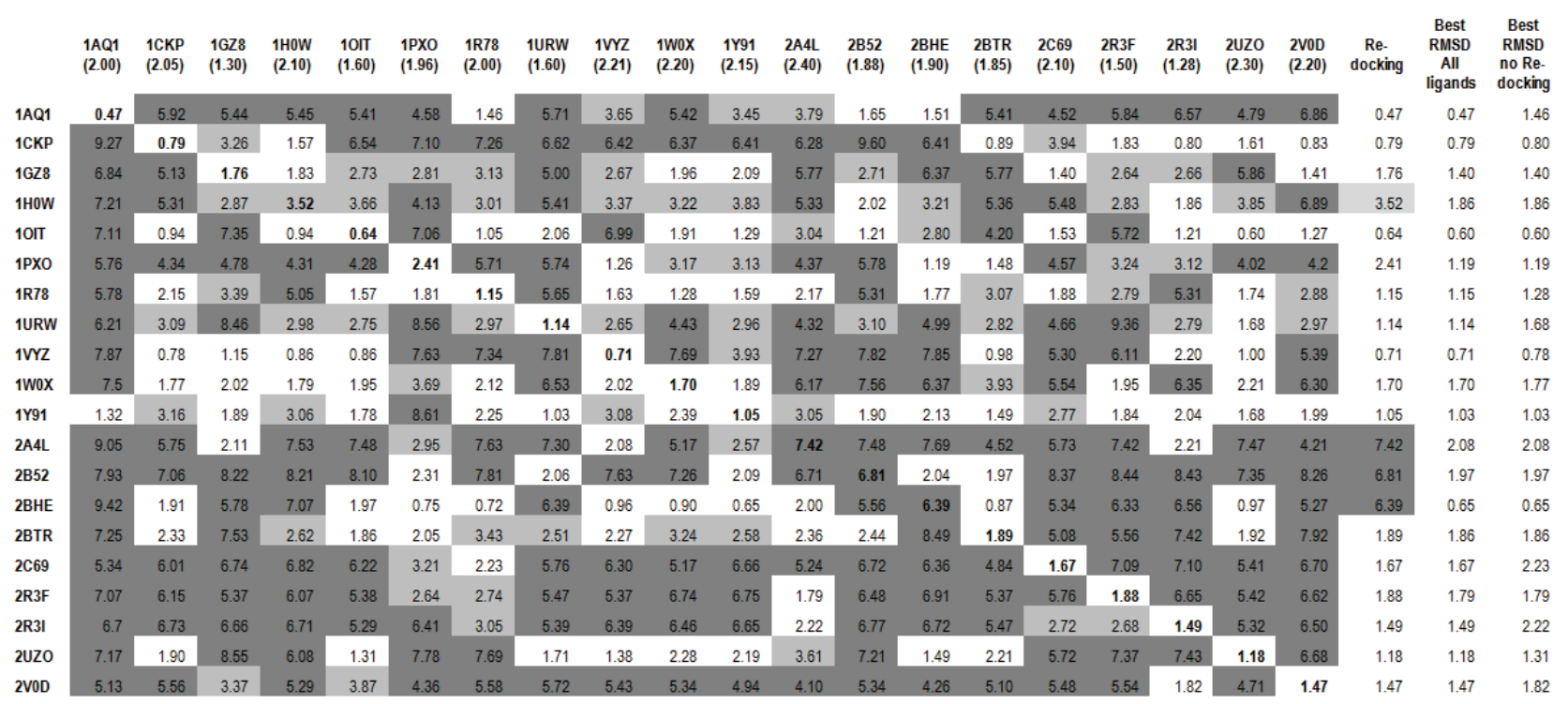

\subsection{Virtual Screening}

A molecular constraint search in the ZINC database based on physico-chemical features of $\mathbf{2 7 n}$, 41n, 42n and 43n compounds resulted in 54,558 Lipinski [20] compliant hits. Before performing docking runs on these compounds, it was observed that the four diverse CDK2 inhibitors (Figure 1) are within $55.16-64.88 \mathrm{kcal} / \mathrm{mol}$ range (Table 1 ) and hence any molecule from the dataset that resulted in scores higher than the range are considered more appropriate. Therefore, in the first step, docking of dataset resulted in 392 hits with dock scores between $41.71-82.33 \mathrm{kcal} / \mathrm{mol}$. In the second step, two approaches were applied to filter the dataset. 


\subsection{Filtering Dataset}

In the first approach, specificity studies between CDK2 and GSK-3 $\beta$ were carried out to filter compounds based on docked energies. All the 392 compounds are docked within the active site regions of $1 \mathrm{UV} 5$ and CDK2 and the data corresponding to the binding energy values of 17 compounds, more specific towards CDK2 after excluding compounds with weak affinity against CDK2 and those that displayed high affinity against GSK-3 $\beta$, are selected.

In the next approach, a consensus scoring was applied, further, to filter these 17 compounds. Initially, the X-Score program was applied to re-evaluate the 17 docked poses. X-Score combines three empirical functions that include van der Waals interaction, hydrogen bonding, deformation penalty and hydrophobic effect. Further scoring was done using eHiTS_score function and each molecule was optimized using eHiTS_score optimization routine to remove steric clashes or other artifacts. In MolDock score of Molegro software, optimization was applied to each molecule using PLP (Piecewise Linear Potential) scoring functions, originally proposed by Gehlhaar et al. [21] and later extended by Yang et al. [22]. Similarly, re-scoring of 17 best docked poses using GOLD score and CHEM score functions are presented in Table 3. In all the cases, RMSD (Root Mean Square Deviation) for all the molecules were within $1 \AA$.

Table 3. Re-scoring and rank-sum data of 17 compounds

\begin{tabular}{c|ccccc|ccccc|c}
\hline & \multicolumn{7}{|c|}{ Score (kcal/mol) } & \multicolumn{5}{c|}{ Rank } & \multirow{2}{*}{$\begin{array}{c}\text { Rank- } \\
\text { Sum }\end{array}$} \\
\cline { 2 - 10 } ZINC ID & eHiTS & GOLD & CHEM & XScore & Molegro & eHiTs & GOLD & CHEM & XScore & Molegro \\
\hline ZINC00695241 & 6.33 & 81.71 & 20.96 & 10.10 & 173.51 & 3 & 3 & 3 & 3 & 3 & 15 \\
ZINC00650519 & 5.75 & 72.20 & 17.73 & 9.96 & 154.76 & 2 & 2 & 3 & 3 & 3 & 13 \\
ZINC00628537 & 6.06 & 71.57 & 22.01 & 9.51 & 152.81 & 3 & 2 & 3 & 2 & 3 & 13 \\
ZINC00984879 & 6.53 & 80.63 & 16.88 & 8.53 & 134.76 & 3 & 3 & 2 & 1 & 2 & 11 \\
ZINC00724381 & 5.05 & 71.96 & 19.38 & 10.51 & 112.09 & 2 & 2 & 3 & 3 & 1 & 11 \\
ZINC00646178 & 5.55 & 72.31 & 17.25 & 9.70 & 140.82 & 2 & 2 & 2 & 3 & 2 & 11 \\
ZINC00686654 & 4.20 & 74.99 & 14.37 & 9.19 & 141.06 & 1 & 2 & 2 & 2 & 2 & 9 \\
ZINC00694967 & 6.86 & 71.13 & 12.11 & 9.42 & 124.59 & 3 & 1 & 1 & 2 & 1 & 8 \\
ZINC00695587 & 4.91 & 70.98 & 17.89 & 8.88 & 146.23 & 1 & 1 & 3 & 1 & 2 & 8 \\
ZINC00624580 & 6.56 & 76.05 & 11.61 & 8.68 & 112.34 & 3 & 2 & 1 & 1 & 1 & 8 \\
ZINC00643387 & 4.09 & 66.36 & 20.15 & 8.56 & 116.14 & 1 & 1 & 3 & 1 & 1 & 7 \\
ZINC01003046 & 4.11 & 68.38 & 8.90 & 9.01 & 111.21 & 1 & 1 & 1 & 2 & 1 & 6 \\
ZINC00899523 & 4.20 & 66.32 & 11.14 & 8.45 & 125.64 & 1 & 1 & 1 & 1 & 1 & 5 \\
ZINC00710353 & 4.77 & 68.54 & 7.85 & 8.17 & 124.54 & 1 & 1 & 1 & 1 & 1 & 5 \\
ZINC00977556 & 4.04 & 68.29 & 8.25 & 8.88 & 110.82 & 1 & 1 & 1 & 1 & 1 & 5 \\
ZINC00994710 & 4.04 & 66.96 & 8.14 & 8.26 & 112.09 & 1 & 1 & 1 & 1 & 1 & 5 \\
ZINC00636674 & 4.01 & 66.15 & 7.89 & 8.08 & 109.19 & 1 & 1 & 1 & 1 & 1 & 5 \\
\hline
\end{tabular}


In all the above cases, ranking was done individually by clustering scores into equally split four classes using Tsar software [www.accelrys.com], of which compounds in Class4 represents the highest class or top rank. Classes were generated for all scoring functions and instead of taking an average, rank-sum technique [23] was employed to retrieve best compounds. The ranks obtained from each of the scoring functions considered in the study were added to give the rank-sum. The advantage of a sum over an average was that the contribution from the rank for each individual score can more easily be split out for illustrative purposes in the former instance. On the other hand, taking cross-docking study into consideration, it should be noted that the single conformation of the CDK2 protein may not be sufficient in screening thousands of compounds and hence all the twenty CDK2 structures reported in Table 2 were selected to evaluate the affinity of binding of 17 compounds obtained after filtering the dataset. The data presented in Table 4 suggests similar affinities and minimal variation in binding of these compounds towards CDK2.

Table 4. Binding affinity data ( $\mathrm{kcal} / \mathrm{mol})$ of 17 compounds against $20 \mathrm{CDK} 2$ protein structures used in the cross-docking study.

\begin{tabular}{|c|c|c|c|c|c|c|c|c|c|c|c|c|c|c|c|c|c|c|c|c|}
\hline \multirow{2}{*}{ ZINC Id } & \multicolumn{20}{|c|}{ Binding Affinities (kcal/mol) } \\
\hline & IAQ1 & ICKP & 1GZ8 & lHow & 10IT & IPXO & IR78 & IURW & IVYZ & 1w0x & $1 Y 91$ & 2A4L & 2B52 & 2BHE & 2BTR & $2 \mathrm{C} 69$ & $2 \mathrm{R} 3 \mathrm{~F}$ & 2R3I & $2 \mathrm{UZO}$ & 2V0D \\
\hline ZINC00695241 & 83.51 & 80.21 & 81.65 & 81.01 & 82.54 & 83.45 & 82.32 & 81.82 & 85.24 & 86.54 & 82.94 & 84.45 & 83.94 & 85.54 & 87.21 & 81.25 & 83.29 & 86.02 & 81.71 & 81.9 \\
\hline ZINC00650519 & 70.76 & 74.65 & 71.41 & 70.15 & 72.51 & 72.65 & 71.34 & 73.23 & 74.66 & 71.25 & 69.23 & 70.53 & 72.44 & 73.79 & 67.64 & 71.87 & 69.54 & 72.66 & 72.2 & 70.19 \\
\hline ZINC00628537 & 72.81 & 72.1 & 70.85 & 72.31 & 71.52 & 72.11 & 73.2 & 70.35 & 74.24 & 75.31 & 69.64 & 71.26 & 72.29 & 73.64 & 76.76 & 70.32 & 74.84 & 77.36 & 71.57 & 75.26 \\
\hline ZINC00984879 & 80.76 & 78.09 & 79.78 & 79.68 & 82.87 & 79.84 & 81.06 & 79.85 & 82.87 & 82.82 & 80.55 & 81.82 & 78.28 & 79.39 & 83.83 & 76.25 & 80.49 & 81.91 & 80.63 & 84.92 \\
\hline ZINC00724381 & 60.09 & 71.12 & 68.15 & 66.15 & 63.1 & 61.54 & 65.23 & 69.47 & 72.58 & 68.45 & 67.74 & 69.21 & 70.55 & 66.84 & 71.94 & 67.66 & 72.44 & 72.96 & 71.96 & 68.63 \\
\hline ZINC00646178 & 68.82 & 72.85 & 76.12 & 72.15 & 73.65 & 70.23 & 75.52 & 71.33 & 75.44 & 74.74 & 72.46 & 73.57 & 70.29 & 71.89 & 68.67 & 67.24 & 73.93 & 75.67 & 72.31 & 69.37 \\
\hline ZINC00686654 & 72.06 & 69.45 & 71.35 & 72.92 & 73.46 & 71.88 & 73.65 & 70.54 & 71.84 & 73.54 & 71.39 & 72.49 & 71.36 & 74.36 & 76.8 & 71.19 & 73.59 & 75.56 & 74.99 & 76.26 \\
\hline ZINC00694967 & 66.59 & 72.08 & 70.95 & 68.35 & 67.42 & 68.34 & 69.24 & 70.64 & 71.87 & 67.57 & 68.67 & 69.79 & 71.01 & 70.44 & 72.09 & 67.21 & 66.62 & 69.09 & 71.13 & 64.83 \\
\hline ZINC00695587 & 71.23 & 70.18 & 66.14 & 67.54 & 69.52 & 70.25 & 68.59 & 72.89 & 72.24 & 74.85 & 69.46 & 73.93 & 70.59 & 71.64 & 74.91 & 68.96 & 66.22 & 72.56 & 70.98 & 69.73 \\
\hline ZINC00624580 & 74.34 & 72.25 & 71.45 & 72.12 & 74.05 & 73.05 & 72.05 & 70.25 & 73.54 & 71.67 & 75.38 & 74.29 & 72.21 & 71.11 & 73.16 & 69.06 & 76.43 & 79.63 & 76.05 & 75.28 \\
\hline ZINC00643387 & 64.14 & 66.89 & 67.2 & 66.07 & 65.15 & 64.33 & 63.76 & 62.88 & 61.52 & 63.84 & 62.45 & 60.89 & 64.65 & 61.36 & 65.19 & 59.09 & 61.91 & 63.42 & 66.36 & 61.2 \\
\hline ZINC01003046 & 67.54 & 69.02 & 67.55 & 65.6 & 63.51 & 61.58 & 66.93 & 65.49 & 66.36 & 63.24 & 65.69 & 64.54 & 67.84 & 66.72 & 68.34 & 69.25 & 67.89 & 70.21 & 68.38 & 68.85 \\
\hline ZINC00899523 & 63.64 & 66.17 & 60.35 & 62.48 & 64.52 & 62.48 & 63.46 & 59.75 & 65.78 & 65.85 & 61.39 & 63.94 & 62.33 & 64.24 & 66.94 & 62.64 & 63.46 & 65.97 & 66.32 & 64.54 \\
\hline ZINC00710353 & 66.54 & 65.35 & 67.41 & 65.34 & 66.65 & 64.32 & 65.51 & 68.38 & 69.86 & 64.97 & 67.83 & 65.39 & 66.54 & 65.72 & 68.64 & 63.99 & 62.89 & 69.63 & 68.54 & 64.64 \\
\hline ZINC00977556 & 66.82 & 68.1 & 68.87 & 67.54 & 68.5 & 69.4 & 67.08 & 65.64 & 69.34 & 64.35 & 67.06 & 65.97 & 66.01 & 68.24 & 69.55 & 65.91 & 67.66 & 70.26 & 68.29 & 64.91 \\
\hline ZINC00994710 & 63.09 & 66.72 & 69.65 & 67.87 & 66.05 & 62.1 & 65.31 & 64.34 & 65.25 & 64.88 & 67.63 & 66.89 & 68.79 & 65.65 & 69.03 & 63.29 & 66.05 & 68.97 & 66.96 & 62.09 \\
\hline ZINC00636674 & 64.19 & 65.92 & 65.45 & 65.85 & 63.12 & 61.82 & 62.65 & 63.99 & 65.24 & 66.12 & 67.22 & 71.38 & 69.46 & 64.45 & 67.21 & 63.58 & 67.4 & 67.54 & 66.15 & 64.27 \\
\hline
\end{tabular}

\subsection{Binding Modes of top three compounds}

Active site of CDK2 offers many different binding modes for inhibitors as they are strongly dependent on the attached substituent. All 39 compounds were docked deeply within the ATP binding pocket region forming hydrogen bond interactions with Lys33, Glu81, Leu83, Asp86, Lys89, Gln131 and Asn132.

ZINC00695241, the first best compound exhibited Leu83, Lys33 and Asn132 hydrogen bond interactions. The $\mathrm{N}$ atom adjacent to the $[1,2,4]$-triazole moiety donates a hydrogen bond to the backbone carbonyl of Leu83. The oxygen atom on ligand accepts hydrogen bond from side chain 
NZ atom of Lys33. Similarly, the O atom on furan ring accepts a hydrogen bond from the side chain atom ND2 of Asn132 (Figure 2).

The next best compound ZINC00650519 formed three H-bonds with Asn132 and Asp145 residues. Similarly ZINC00628537 displayed three H-bond interactions with Leu83, Lys129 and Asn132 (Figures 3 and 4). The hydrogen bond interaction of top ten compounds is given in Table 5 and the majority of H-bond interactions formed by these compounds are: Leu83, Asn132, and Asp145 respectively.

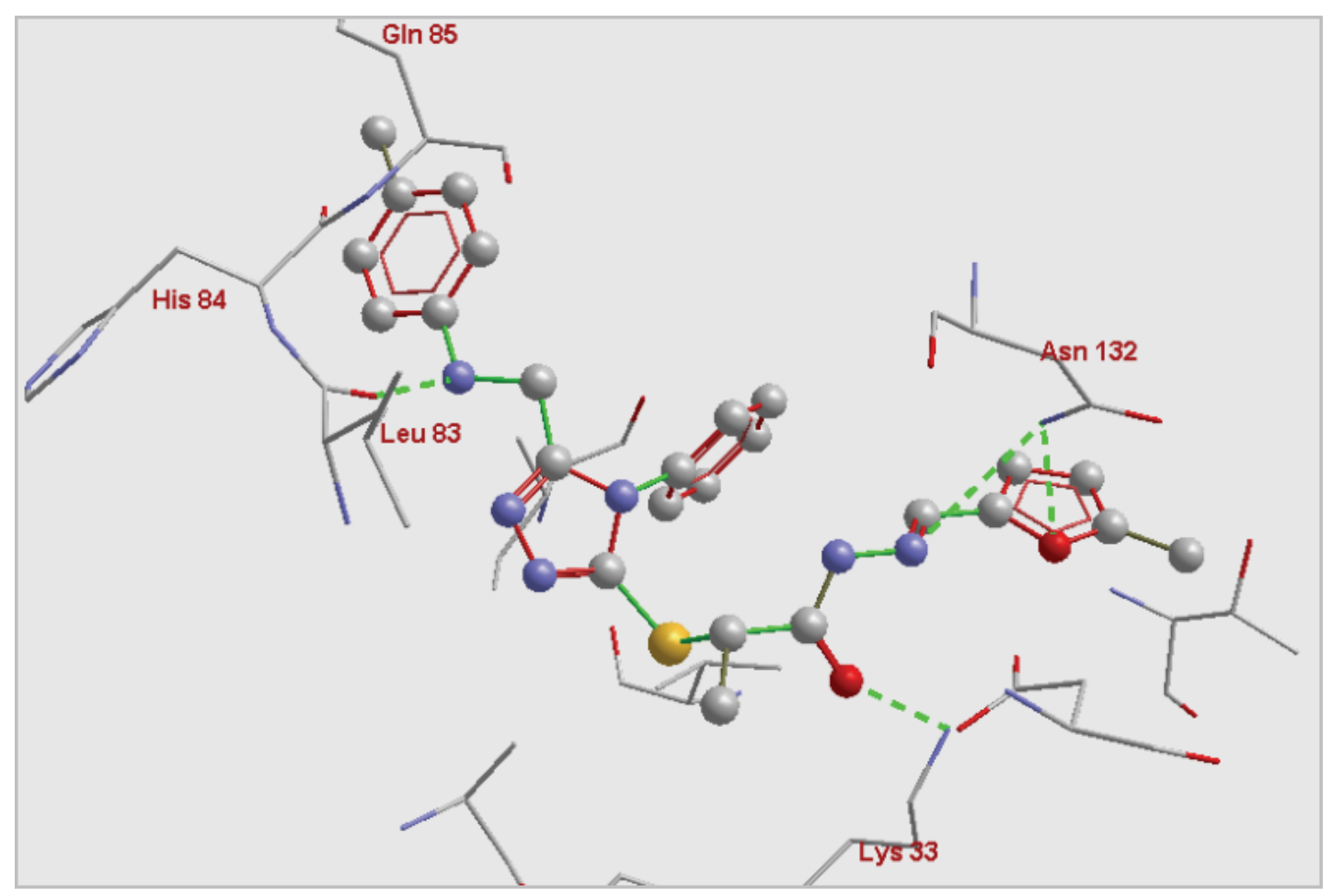

Figure 2. Active site region of 2UZO complexed with ZINC00695241

Hydrogen bonds with Lys33, Leu83 and Asn132 are depicted as green dashed lines. 


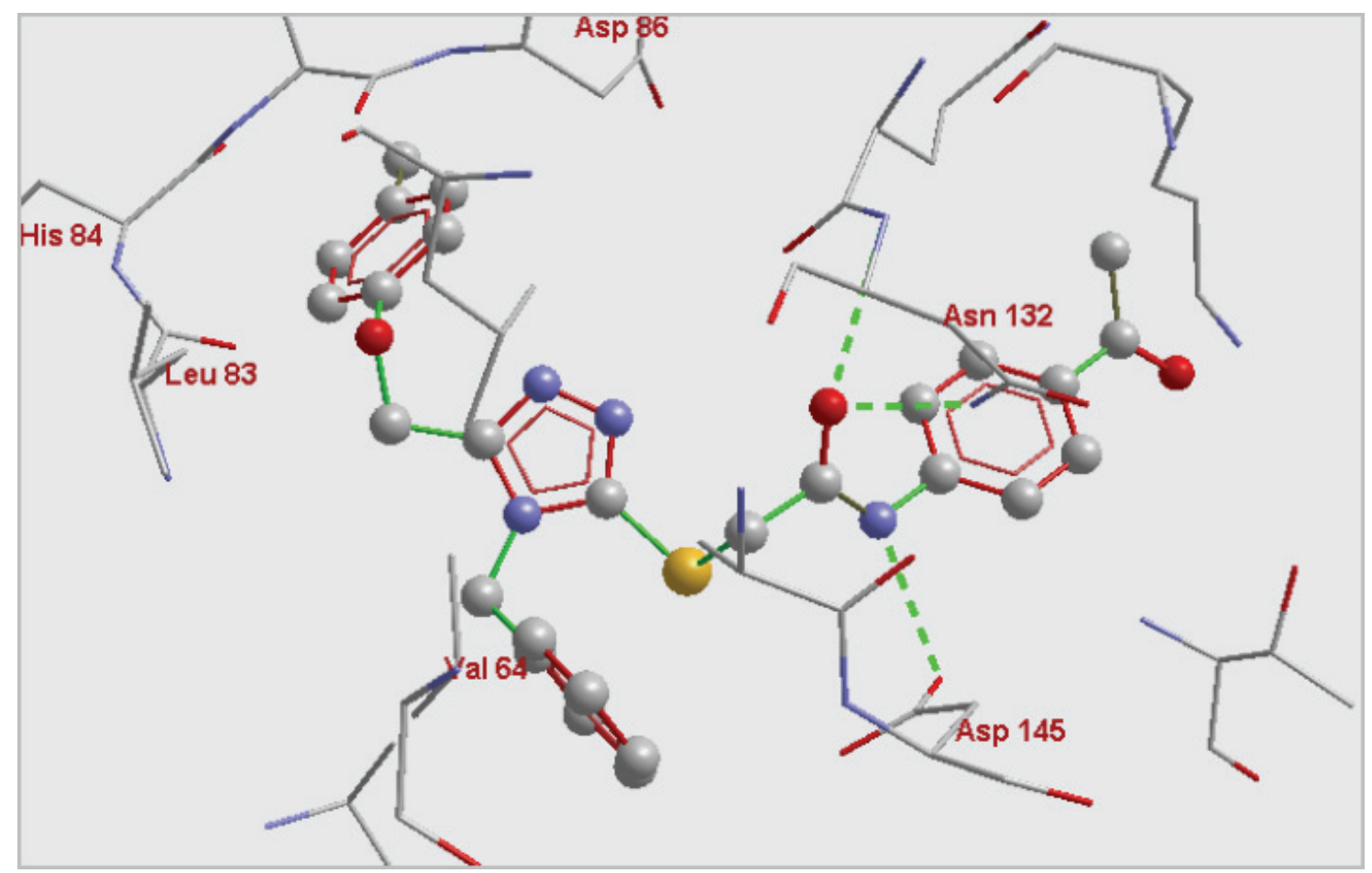

Figure 3. Active site region of 2 UZO complexed with ZINC00650519

Hydrogen bonds with Asn132 and Asp145 are depicted as green dashed lines.

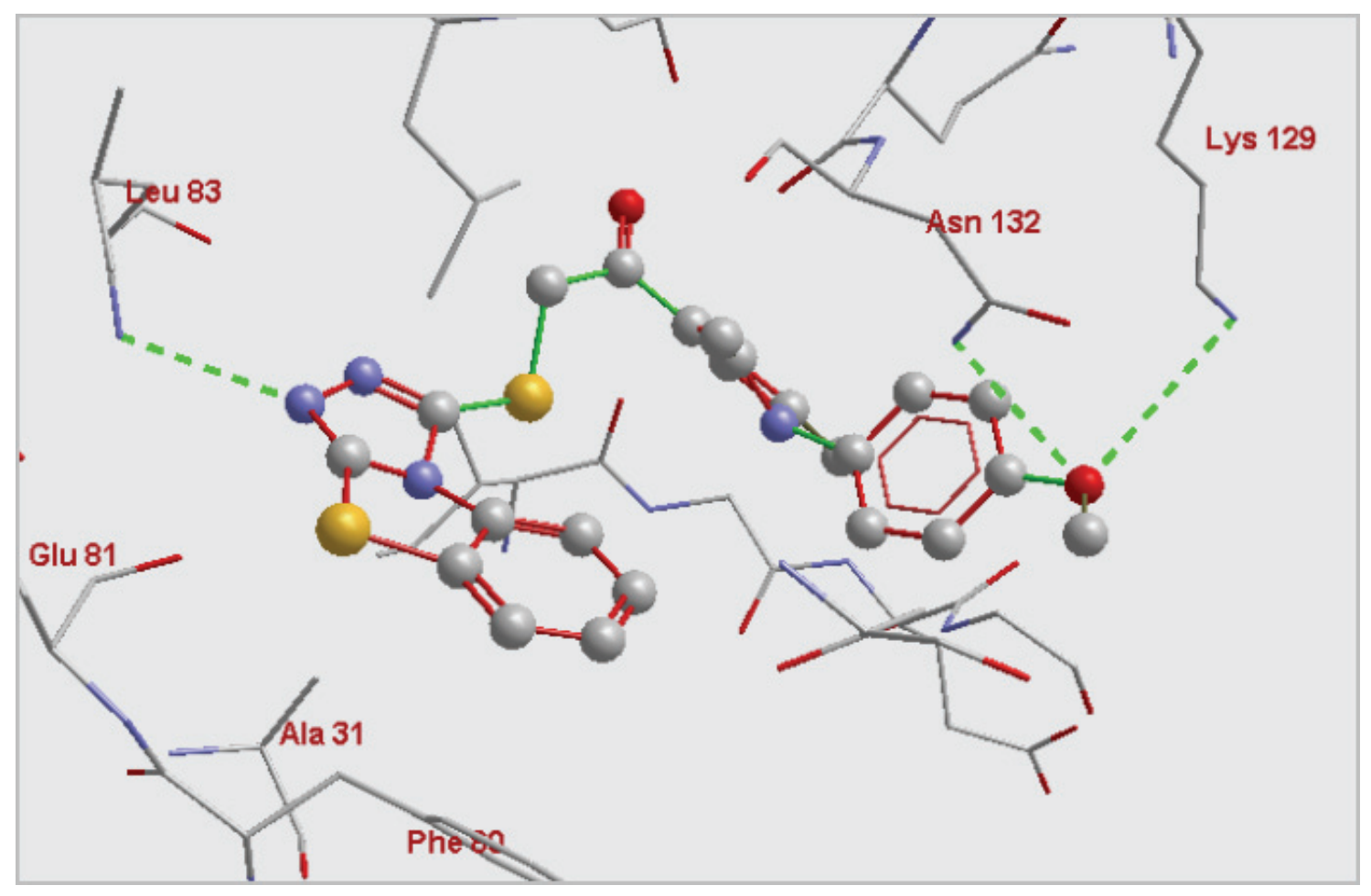

Figure 4. Active site region of $2 \mathrm{UZO}$ complexed with ZINC00628537

Hydrogen bonds with Leu83, Lys129 and Asn132 are depicted as green dashed lines. 
Table 5. H-bond interacting residues of top ten ZINC compounds

\begin{tabular}{ll}
\hline ZINC Id & H-bond interacting residues \\
\hline ZINC00695241 & Leu83, Lys33, Asn132 \\
ZINC00650519 & Asn132, Asp145 \\
ZINC00628537 & Leu83, Lys129, Asn132 \\
ZINC00984879 & Leu83, His84, Asp86, Gln131 \\
ZINC00724381 & Ile10, Asn132, Asp145 \\
ZINC00646178 & Glu81, Leu83 \\
ZINC00686654 & Leu83 \\
ZINC00694967 & Lys33, Leu83, Asp145 \\
ZINC00695587 & Leu83, Asp145 \\
ZINC00624580 & Glu81,Leu83 \\
\hline
\end{tabular}

Finally, cell based assay was selected as another screening technique to filter compounds that exhibit inhibitory activity $\left(\mathrm{IC}_{50}\right)$. Compounds with high $\log \mathrm{P}$ values enhance their ability to penetrate lipid membranes by passive diffusion, thereby enhancing their potential for absorption. Moreover, compounds with negative $\log \mathrm{P}$ values penetrate less than positive through cell membranes [24]. All compounds are within 4.25 to $4.95 \log P$ values, which refer to the lipophilic nature and are related to their ability to cross biological membranes. Therefore, from a dataset of 17 compounds, the top ten compounds (Table 6) were examined for anti-proliferative activity against human lung adenocarcinoma epithelial cell line, A549 using MTT assay.

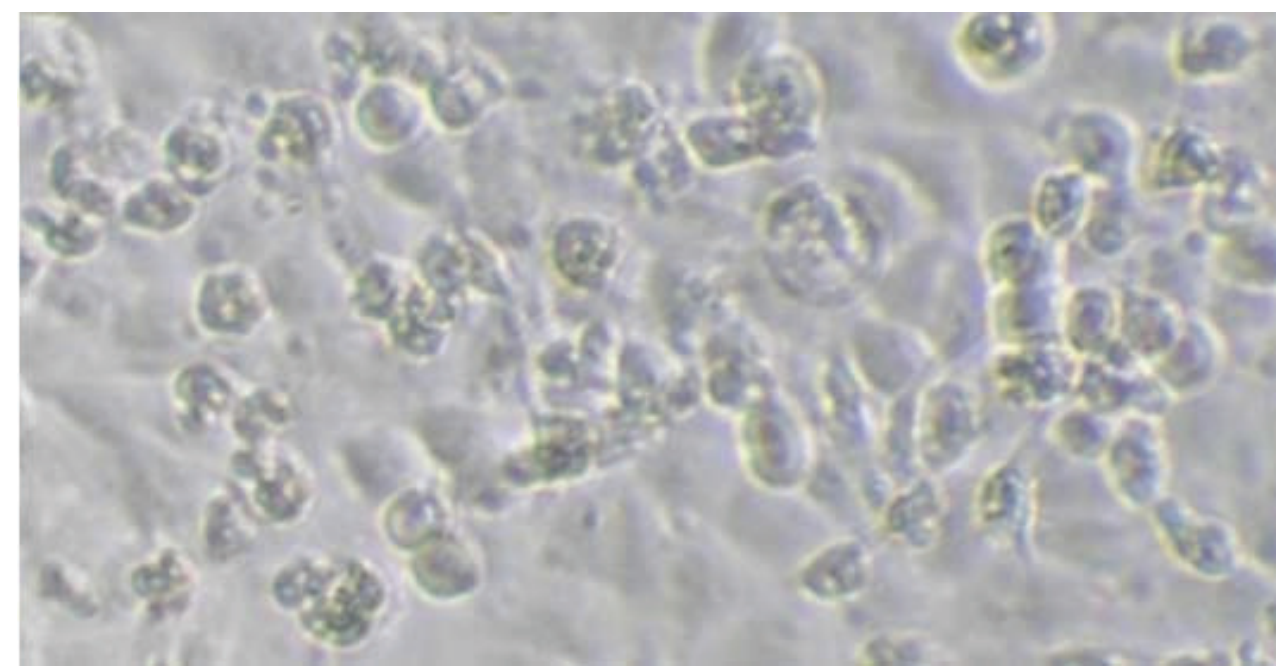

Figure 5. Apoptotic cell death of A549 cell line when treated with compound 2 
The $\mathrm{IC}_{50}$ values were between 5.1 - $60.7 \mu \mathrm{mol} / \mathrm{L}$ (Table 6) and a gradual decrease in the viability of A549 cells were observed in a dose-dependent manner for all the compounds under study. At the highest concentration of the tested dose $(64 \mu \mathrm{g} / \mathrm{ml})$, the maximum rate of inhibition observed was $87.94 \%$ for compound 4. Compound 2 with $[1,2,4]$ triazol-3-yl sulfanyl moiety was found to be the most active with $\mathrm{IC}_{50}$ value $5.1 \mu \mathrm{mol} / \mathrm{L}$. The morphology of the cells after treatment with compounds was found to be apoptotic, characterized by apoptotic bodies (Figure 5). It has been reported in literature that [1,2,4]triazol analogs are potent anti-cancer compounds [25][26], and hence $[1,2,4]$ triazol-3-yl sulfanyl substituted compounds reported in this work represent a new scaffold for novel anti-cancer ligand design. Moreover, it is worthwhile to note a comparison between compounds $\mathbf{8}$ and $\mathbf{9}$, where they differ at 4-position substitution on the $[1,2,4]$ triazol-3-yl sulfanyl basic scaffold. The 4-allyl substituted compound 9 has high anti-proliferative activity ( $\mathrm{IC}_{50}$ : $13 \mu \mathrm{mol} / \mathrm{L})$ than the 4-phenyl substituted compound 8 ( $\left.\mathrm{IC}_{50}: 25.2 \mu \mathrm{mol} / \mathrm{L}\right)$. Further exploration of these compounds at 4-position would certainly reveal important aspects relevant to this feature. On the other hand, a thiourea with oxazolo[4,5-b]pyridine substituted compound 5 showed high antiproliferative effect and the concentration required for effective inhibition was found to be 6.0 $\mu \mathrm{mol} / \mathrm{L}$. 
Table 6. $\mathrm{IC}_{50}$ values of final set of ten compounds screened for anti-proliferative activity against A549 cell line.

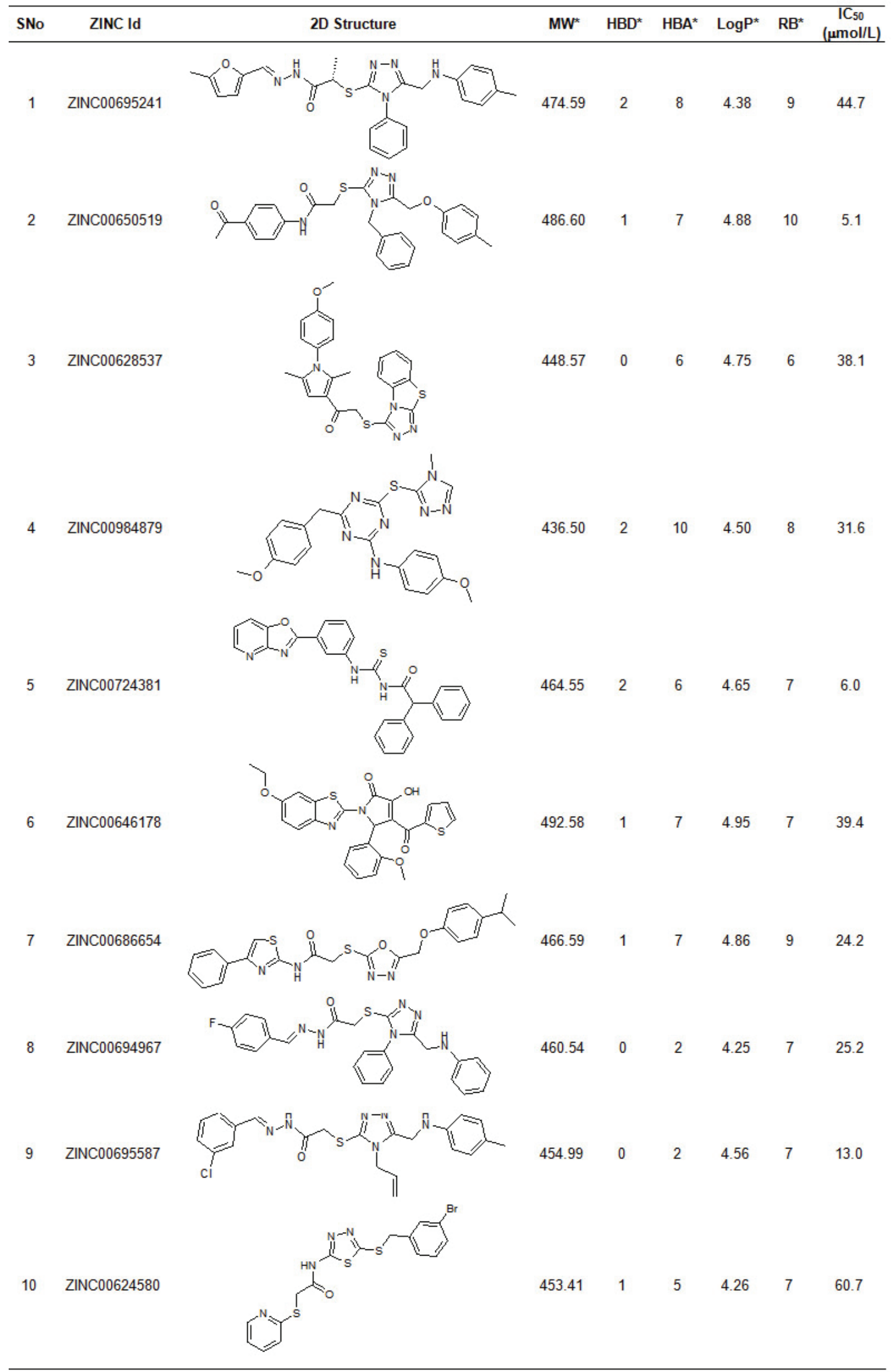


Finally, this study clearly demonstrated the importance of docking studies in screening compound libraries and illustrated the use of simple, yet robust filter techniques that guided us to uncover entirely novel compounds from a huge dataset. To the best of our knowledge, these specific compounds have not been reported as anti-cancer agents so far in the literature.

\section{Conclusion}

Our study has identified novel anti-cancer agents and the approach based on computer-aided drug design technique resulted in an entirely diverse range of CDK2 inhibitors from ZINC database. Docked poses of various ligands within the active site region of CDK2 revealed a remarkable structural diversity with significant binding orientations. The final data set of filtered compounds was investigated for their ability to inhibit A549 cell proliferation using MTT assay. Nearly three compounds showed moderate growth inhibitory effect with $\mathrm{IC}_{50}$ values below $13.0 \mu \mathrm{mol} / \mathrm{L}$ on apoptosis of A549 cells. Therefore these newly discovered compounds from virtual screening analysis provides a novel scaffold for further design and development of potent anti-cancer agents.

\section{References}

[1] A.W. Murray, Recycling the cell cycle: cyclins revisited, Cell, 116, 221-234 (2004).

[2] M. Hatakeyama and R.A. Weinberg, The role of RB in cell cycle control, Prog Cell Cycle Res, 1, 9-19 (1995).

[3] S. Grant and J.D. Roberts, The use of cyclin-dependent kinase inhibitors alone or in combination with established cytotoxic drugs in cancer chemotherapy, Drug Resist Updat, 6, 15-26 (2003).

[4] T.G. Davies, D.J. Pratt, J.A. Endicott, L.N. Johnson and M.E. Noble, Structure-based design of cyclin-dependent kinase inhibitors, Pharmacol Ther, 93, 125-133 (2002).

[5] P.A. Babu, M.L. Narasu and K. Srinivas, Pyridines, pyridazines and guanines as CDK2 inhibitors: a review, Arkivoc, ii, 247-265 (2007).

[6] J.J. Irwin and B.K. Shoichet, ZINC--a free database of commercially available compounds for virtual screening, J Chem Inf Model, 45, 177-182 (2005).

[7] P.A. Babu, D.J. Smiles, M.L. Narasu and K. Srinivas, Identification of Novel CDK2 Inhibitors by QSAR and Virtual Screening Procedures, QSAR \& Comb Sci, 27, 1362-1373 (2008). 
[8] C.M. Richardson, C.L. Nunns, D.S. Williamson, M.J. Parratt, P. Dokurno, R. Howes, J. Borgognoni, M.J. Drysdale, H. Finch, R.E. Hubbard, P.S. Jackson, P. Kierstan, G. Lentzen, J. D. Moore, J. B. Murray, H. Simmonite, A.E. Surgenor and C.J. Torrance, Discovery of a potent CDK2 inhibitor with a novel binding mode, using virtual screening and initial, structure-guided lead scoping, Bioorg Med Chem Lett, 17, 3880-3885 (2007).

[9] G. Jones, P. Willett, R.C. Glen, A.R. Leach and R. Taylor, Development and validation of a genetic algorithm for flexible docking, J Mol Biol, 267, 727-748 (1997).

[10] D.B. Kitchen, H. Decornez, J.R. Furr and J. Bajorath, Docking and scoring in virtual screening for drug discovery: methods and applications, Nat Rev Drug Discov, 3, 935-949 (2004).

[11] R. Wang, Y. Lu and S. Wang, Comparative Evaluation of 11 Scoring Functions for Molecular Docking, J Med Chem, 46, 2287-2303 (2003).

[12] P.S. Charifson, J.J. Corkery, M.A. Murcko and W.P. Walters, Consensus Scoring: A Method for Obtaining Improved Hit Rates from Docking Databases of Three-Dimensional Structures into Proteins, J Med Chem, 42, 5100-5109 (1999).

[13] Z. Zsoldos, D. Reid, A. Simon, S.B. Sadjad and A.P. Johnson, eHiTS: a new fast, exhaustive flexible ligand docking system, J Mol Graph Model, 26, 198-212 (2007).

[14] R. Thomsen and M.H. Christensen, MolDock: a new technique for high-accuracy molecular docking, J Med Chem, 49, 3315-3321 (2006).

[15] R. Wang, L. Lai and S. Wang, Further development and validation of empirical scoring functions for structure-based binding affinity prediction, J Comput Aided Mol Des, 16, 11-26 (2002).

[16] T. Mosmann, Rapid colorimetric assay for cellular growth and survival: Application to proliferation and cytotoxicity assays, J Immunol Methods, 65, 55-63 (1983).

[17] P.K. Gadakar, S. Phukan, M.D. Prasanna and V.N. Balaji, Enrichment of potent GSK-3b Inhibitors from docking studies in the enzyme active site, Current Sci, 93, 1100-1107 (2007).

[18] P.K. Gadakar, S. Phukan, M.D. Prasanna and V.N. Balaji, Pose prediction accuracy in docking studies and enrichment of actives in the active site of GSK-3beta, J Chem Inf Model, 47, 1446-1459 (2007).

[19] V.A. Ilyin, A. Abyzov and C.M. Leslin, Structural alignment of proteins by a novel TOPOFIT method, as a superimposition of common volumes at a topomax point, Protein Sci, 13, 18651874 (2004).

[20] C.A. Lipinski, Drug-like properties and the causes of poor solubility and poor permeability, $J$ Pharmacol Toxicol Methods, 44, 235-249 (2000). 
[21] D.K. Gehlhaar, G. Verkhivker, P.A. Rejto, D.B. Fogel, L.J. Fogel and S.T. Freer, Docking Conformationally Flexible Small Molecules into a Protein Binding Site through Evolutionary Programming, In: J.R. McDonnell, R.G. Reynolds, D.B. Fogel (Eds.) Proceedings of the Fourth Annual Conference on Evolutionary Programming, MIT Press, Cambridge, Massachusetts, 615-627 (1995).

[22] J.M. Yang and C.C. Chen, GEMDOCK: A Generic Evolutionary Method for Molecular Docking, Proteins, 55, 288-304 (2004).

[23] R.D. Clark, A. Strizhev, J.M. Leonard, J.F. Blake and J.B. Matthew, Consensus scoring for ligand/protein interactions, J Mol Graph Model, 20, 281-295 (2002).

[24] C.A. Lipinski, F. Lombardo, B.W. Dominy and P.J. Feeney, Experimental and computational approaches to estimate solubility and permeability in drug discovery and development settings, Adv Drug Deliv Rev, 23, 3-25 (1997).

[25] A.S. Formagio, L.T. Tonin, M.A. Foglio, C. Madjarof, J.E. de Carvalho, W.F. da Costa, F.P. Cardoso and M. H. Sarragiotto, Synthesis and antitumoral activity of novel 3-(2-substituted1,3,4-oxadiazol-5-yl) and 3-(5-substituted-1,2,4-triazol-3-yl) $\beta$-carboline derivatives, Bioorg Med Chem, 16, 9660-9667 (2008).

[26] R. Lesyk, O. Vladzimirska, S. Holota, L. Zaprutko and A. Gzella, New 5-substituted thiazolo[3,2-b][1,2,4]triazol-6-ones: synthesis and anticancer evaluation, Eur J Med Chem, 42, 641-648 (2007). 